\title{
Experience with pegvisomant treatment in acromegaly in a single Brazilian tertiary reference center: efficacy, safety and predictors of response
}

Leandro Kasuki ${ }^{1,2,3}$, Evelyn de Oliveira Machado', Liana Lumi Ogino², Maria Caroline Alves Coelho ${ }^{1,4,5}$, Cintia Marques dos Santos Silva', Luiz Eduardo Armondi Wildemberg ${ }^{1,2}$, Carlos Henrique Azeredo Lima ${ }^{2}$, Mônica R. Gadelha',2

\begin{abstract}
Objective: To describe the safety and efficacy of pegvisomant therapy and the predictors of treatment response in acromegaly patients at a single tertiary reference center in Brazil. Materials and methods: We retrospectively reviewed the clinical, hormonal and radiological data of acromegaly patients treated with pegvisomant in our center. We also evaluated the presence of the $\mathrm{d} 3$ isoform of the growth hormone receptor (d3GHR). Results: Twenty-seven patients were included (17 women). Pegvisomant was used in combination with octreotide LAR in 20 patients (74\%), in combination with cabergoline in one $(4 \%)$ and as monotherapy in six (22\%). IGF-I normalization was achieved in 23 patients (85\%). Mild and transitory elevation of liver enzymes was observed in two patients $(7.4 \%)$, tumor growth in one $(3.4 \%)$ and lipodystrophy in two $(7.4 \%)$. One patient stopped the drug due to headaches. The GHR isoforms were evaluated in 14 patients, and the presence of at least one d3GHR allele was observed in $43 \%$ of them, but it was not a predictor of treatment response. Only pretreatment IGF-I level was a predictor of treatment response. Conclusion: Pegvisomant treatment was highly effective and safe in our series of Brazilian patients. A better chance of disease control can be expected in those with lower pre-pegvisomant IGF-I levels. Arch Endocrinol Metab. 2016;60(5):479-85
\end{abstract}

Keywords

Acromegaly; pegvisomant; growth hormone isoform
1 Faculdade de Medicina, Centro de Pesquisa em Neuroendocrinologia, Serviço de Endocrinologia, Hospital Universitário Clementino Fraga Filho, Universidade Federal do Rio de Janeiro (HUCFF/UFRJ), Rio de Janeiro, RJ, Brasil

2 Serviço de Neuroendocrinologia, Laboratório de Genética Molecular, Instituto Estadual do Cérebro Paulo Niemeyer, Rio de Janeiro, RJ, Brasil ${ }^{3}$ Serviço de Endocrinologia, Hospital Federal de Bonsucesso, Rio de Janeiro, RJ, Brasil ${ }^{4}$ Serviço de Endocrinologia, Hospital Pedro Ernesto, Rio de Janeiro, RJ, Brasil ${ }^{5}$ Serviço de Endocrinologia, Instituto Estadual de Diabetes e Endocrinologia Luiz Capriglione (IEDE), Rio de Janeiro, RJ, Brasil

Correspondence to: Mônica R. Gadelha

Rua Prof. Rodolpho Paulo Rocco, 255, $9^{\circ}$ andar, Ilha do Fundão,

21941-913 - Rio de

Janeiro, RJ, Brasil

mgadelha@hucff.ufrj.br

Received on: Jan/22/2016

Accepted on: May/18/2016

DOI: $10.1590 / 2359-3997000000210$

\section{INTRODUCTION}

A cromegaly is a rare disease resulting from hypersecretion of growth hormone $(\mathrm{GH})$ and as a consequence of insulin like growth factor-I (IGF-I), which in most cases is caused by a GH-secreting pituitary adenoma (1). Uncontrolled acromegaly is associated with increased morbidity and mortality $(2,3)$. Surgery is the primary treatment in most cases, but approximately half of the patients will not be cured by the surgical procedure and therefore will need adjuvant medical therapy $(4,5)$.

Three drug classes are currently available for acromegaly therapy: somatostatin analogues (SA), dopamine agonists (DA) and GH receptor (GHR) antagonists $(4,6)$. Somatostatin analogues are considered the first option of medical treatment in the majority of patients, but prospective randomized studies show control rates of $20-40 \%$ for patients with first-generation SA (4,7-11). Pasireotide LAR, a next-generation SA (not yet approved for acromegaly treatment in Brazil), allows disease control in a higher percentage of patients and is effective in approximately $15 \%$ of those patients not controlled by first-generation SA with the dose of $40 \mathrm{mg}$ and in $20 \%$ of the patients with the dose of $60 \mathrm{mg}(12,13)$. The efficacy of cabergoline as monotherapy has not been evaluated in prospective studies, but normalization of IGF-I was reported in $34 \%$ of the patients in a meta-analysis of 
the literature, but can be as low as $10 \%$ in more recent studies, therefore, it is reserved for those patients with mildly elevated GH and IGF-I levels (4,14-16). The efficacy of other DA, like bromocriptine, is probably lower than that of cabergoline (4). These two drug classes (SA and DA) act on somatotropinoma through binding to its receptors $(17,18)$.

Pegvisomant is the only drug in the GHR antagonist class and acts by binding to the GHR without triggering its intracellular pathways (19). It can be used in monotherapy or in combination therapy with SA, in this case with greater efficacy. Normalization of IGF-I levels was achieved in more than $90 \%$ of the patients in the initial clinical trials and in $63.2 \%$ of the patients in the last update report of the Acrostudy (20-22). Therefore, it is the most effective drug in controlling IGF-I levels in acromegaly.

There are no robust predictors of the response to pegvisomant treatment, although pre-treatment $\mathrm{GH}$ and IGF-I levels, gender, body mass index, fat mass, presence of type 2 diabetes mellitus (DM), age and previous radiotherapy can influence the chance of disease control $(23,24)$. Additionally, some studies evaluated the GHR polymorphisms as a possible cause of a lower response to the drug, with conflicting results regarding whether patients who present the exon-3 deleted GHR (d3GHR) had a better response to pegvisomant (25-28).

Although an important tool for the management of acromegaly, pegvisomant treatment has never been described in a Brazilian multiethnic population, likely due to the limited availability of the drug in Brazil, considering that the treatment is not reimbursed by the Brazilian government. Therefore, the aim of this study was to describe the safety and efficacy of pegvisomant therapy and predictors of the treatment response in acromegaly patients at a single tertiary reference center in Brazil.

\section{MATERIALS AND METHODS}

\section{Study population}

We retrospectively reviewed the databank of acromegaly patients treated at the endocrinology outpatient clinic of the Hospital Universitário Clementino Fraga Filho (HUCFF), Universidade Federal do Rio de Janeiro (UFRJ) and selected those who were treated with pegvisomant for at least three months.
The diagnosis of acromegaly was made according to clinical and laboratory features, including increased serum IGF-I levels, according to the age and lack of $\mathrm{GH}$ suppression to less than $1.0 \mu \mathrm{g} / \mathrm{L}$ during the $75 \mathrm{~g}$ oral glucose tolerance test (4). Sellar magnetic resonance imaging revealed a pituitary adenoma in all of the patients.

\section{Clinical, hormonal, radiological and treatment data}

We collected the following data: age, sex, GH and IGF-I levels and tumor size at diagnosis and at the beginning of pegvisomant treatment, previous surgery and/or radiotherapy, previous acromegaly treatment, concomitant acromegaly treatment, duration of pegvisomant treatment and maximal pegvisomant dose.

\section{Safety data}

We reviewed data of the MRI and liver enzymes before and during pegvisomant treatment. We also reviewed the possible other side effects related to the drug such as lipodystrophy.

\section{Treatment protocol}

For all patients who are treated with pegvisomant in the endocrinology outpatient clinic of HUCFF/UFRJ, a pituitary MRI is obtained immediately before the beginning of the treatment, and GH and IGF-I levels, liver enzymes (alanine aminotransferase and aspartate aminotransferase) and glucose levels are measured. Pegvisomant is started at a dose of $10 \mathrm{mg} /$ day, and the dose is increased by $5 \mathrm{mg}$ in consecutive increments every month of treatment until normal age-matched IGF-I levels are obtained (4). Somatostatin analogues are maintained if there is a biochemical (at least $20 \%$ of $\mathrm{GH}$ and/or IGF-I reduction) and/or tumor response (tumor stabilization or reduction $>20 \%$ ) with the drug. In the absence of a response to SA, pegvisomant is started as a monotherapy. The dosage of the previous SA treatment is not changed when combination therapy with pegvisomant is initiated. In those patients with concomitant SA treatment and good control with low dose pegvisomant $(10 \mathrm{mg} /$ day $)$, a weekly dose of $60 \mathrm{mg}$ is implemented with a subsequent reduction to the minimal weekly dose, which is sufficient to maintain the IGF-I levels in the mid-range of the normal reference for appropriate age. For safety reasons, liver enzymes are measured monthly for the first six months and then each three months for six months and biannually 
thereafter. The pituitary MRI is repeated after six months of treatment and then annually. Efficacy was evaluated considering the last visit IGF-I level.

\section{Hormone assays}

Plasma GH levels were measured by a chemiluminescence assay kit (IMMULITE 2000; DPC - Diagnostic Products Corp., Inc., Los Angeles, CA, USA). The inter- and intra-assay coefficients of variation $(\mathrm{CV})$ were 6.0 and $5.8 \%$, respectively. The International Reference Preparation (IRP) for GH was 98/574. The plasma IGF-I levels were measured by a chemiluminescence assay kit (IMMULITE 2000; DPC). The inter- and intra-assay CV were 6.6 and $3.6 \%$, respectively (29). The IRP for IGF-I was $87 / 518$. The IGF-I level was expressed as a percentage of the ULNR.

\section{Growth hormone receptor genotyping}

The DNA was extracted from blood leukocytes with the Gentra Puregene Blood Kit (Qiagen, Valencia, CA, USA) according to the manufacturer's protocol, and genotyping of GHR polymorphisms was conducted as follows:

A polymerase chain reaction (PCR) was performed with the following primers: one sense $(\mathrm{Gl}$ : 5'-TGTGCTGGTCTGTTGGTCTG-3') and two antisenses(G2: 5' -AGTCGTTCCTGGGACAGAGA-3' and G3: 5'-CCTGGATTAACACTTTGCAGACTC-3') [GenBank: AF 155912]. Briefly, the PCR was conducted in a $25 \mu \mathrm{L}$ reaction mix using Hotstar Taq DNA polymerase (Qiagen) with denaturation at $94{ }^{\circ} \mathrm{C}$ for $5 \mathrm{~min}$, followed by 35 cycles of $94^{\circ} \mathrm{C}$ for $30 \mathrm{sec}$, $60{ }^{\circ} \mathrm{C}$ for $30 \mathrm{sec}$, and $72{ }^{\circ} \mathrm{C}$ for $90 \mathrm{sec}$, and a final extension phase at $72{ }^{\circ} \mathrm{C}$ for $7 \mathrm{~min}$. Then, the reaction products were run with ethidium bromide-stained $2 \%$ agarose gel electrophoresis. A full-length GHR allele (flGHR) was shown by the presence of two bands of approximately $935 \mathrm{bp}$. In the presence of the genomic deletion of exon 3 (d3GHR), a 532-bp band was formed.

\section{Statistical analysis}

The statistical analyses were performed using SPSS version 20.0 for MacOS (SPSS Inc., Chicago, IL). For the descriptive analysis, categorical variables were expressed as the percentage and frequency, and the numerical variables were expressed as the mean $\pm \mathrm{DP}$ or median $(\min -\max )$ according to the distribution of the sample. The difference between the IGF-I levels before and after pegvisomant therapy was evaluated by the Wilcoxon test. The Spearman test was used for correlations. A p-value $<0.05$ was considered statistically significant.

\section{RESULTS}

\section{Characterization of the study population}

\section{Clinical, biochemical and previous treatment characteristics}

A total of 27 patients [ 17 women (63\%)] were enrolled in the study. The mean age at diagnosis was $41.3 \pm 16.7$ years. Twenty-one patients $(78 \%)$ were submitted to surgery and 11 to radiotherapy (41\%). A macroadenoma was observed during diagnosis in 25 patients $(93 \%)$. Median GH and IGF-I levels during diagnosis were $14.2 \mu \mathrm{g} / \mathrm{L}(3.4-252.0)$ and $295 \%$ ULNR (157 $671)$, respectively. Twelve patients $(44 \%)$ presented diabetes mellitus before pegvisomant treatment.

Octreotide LAR treatment was attempted before pegvisomant in 26 patients (96\%). It was the primary therapy in 6 patients: two patients presented high surgical risk and four patients presented tumors that were mainly located in the cavernous sinus. The maximal dose was $30 \mathrm{mg}$ every 28 days in 16 patients and $40 \mathrm{mg}$ every 28 days in 10 patients. The association of cabergoline to octreotide LAR treatment was also attempted in 21 of the 27 patients (78\%).

\section{Pegvisomant treatment}

\section{Efficacy}

The median GH and IGF-I levels before pegvisomant treatment were $3.7 \mu \mathrm{g} / \mathrm{L}(0.8-209.0)$ and $212 \%$ ULNR (132 - 637), respectively. Pegvisomant was used in combination with octreotide LAR in 20 patients $(74 \%)$ and in combination with cabergoline in one patient $(4 \%)$. The dose of octreotide LAR was $20 \mathrm{mg}$ in one patient and $30 \mathrm{mg}$ in the remaining 19 patients during combination therapy. The dose of cabergoline was $3.0 \mathrm{mg} /$ week in the only patient who used cabergoline in association with pegvisomant. In six patients, pegvisomant was used as single treatment. The median treatment duration was 15 months $(3-$ 69 months), and the median pegvisomant dose was 10 $\mathrm{mg} /$ day [6 (40 mg/week) - $30 \mathrm{mg} /$ day] . 
Acromegaly control (normalization of IGF-I) was obtained in 23 patients (85\%). The normalization of IGF-I was obtained in five out of six patients in monotherapy (83\%) and in 18 out of 21 patients $(86 \%)$ in combination therapy. In three patients, a transition to a weekly dose of PEG was possible (ranging from $40-70 \mathrm{mg} /$ week) with maintenance of normal IGF-I levels. The median IGF-I levels after treatment were $76 \%$ ULNR ( 47 - 308). The IGF-I levels presented a median reduction of $66 \%(0-82)$ from pre-treatment values. The median dose of pegvisomant was 10 $\mathrm{mg} /$ day $(6-20 \mathrm{mg} /$ day $)$ in the controlled patients, whereas it was $22.5 \mathrm{mg} /$ day $(10-30 \mathrm{mg} /$ day $)$ in the uncontrolled patients.

\section{Predictors of response}

There was no difference in the age (at diagnosis or at the moment of pegvisomant initiation), sex, frequency of previous radiotherapy or GH or IGF-I levels at diagnosis between patients who were controlled and those who were not after pegvisomant treatment (Table 1).

The median $\mathrm{GH}$ level before pegvisomant treatment was $2.8 \mu \mathrm{g} / \mathrm{L}(0.7-17.3)$ in those patients controlled after treatment, whereas it was $6.5 \mu \mathrm{g} / \mathrm{L}(2.6-209.0)$ in non-controlled patients, although the difference did not reach statistical significance $(\mathrm{p}=0.095)$. The pretreatment median IGF-I levels were lower in those patients controlled after pegvisomant treatment than in those not controlled after treatment [208\% ULNR $(132-390)$ vs. 559\% ULNR (214-637), $\mathrm{p}=0.004$ ] (Table 1 ).

There was no correlation between the age at diagnosis, pre-treatment GH or IGF-I levels with the percentage of IGF-I reduction after treatment.

Growth hormone receptor polymorphisms were studied in 14 patients. The flGHR was observed in both alleles in eight patients (57\%). Two patients (14\%) were homozygous and four patients (29\%) was heterozygous for the $\mathrm{d} 3 \mathrm{GHR}$ allele. There was no difference in age, GH or IGF-I levels before pegvisomant treatment or in the percentage of IGF-I reduction between homozygous flGHR patients and those who were not. Additionally, there was no difference in the frequency of IGF-I normalization between patients homozygous for the flGHR and those who were not.

\section{Safety}

Tumor enlargement was observed in one patient $(3.7 \%)$, but tumor growth continued despite withdrawal of pegvisomant (Figure 1). This was a young patient with an aggressive tumor since diagnosis (the tumor presented a Ki-67 labeling index of 4.0\% and a p53 of $6.0 \%$ ). The patient was submitted to surgery and treated with octreotide LAR $30 \mathrm{mg}$ every 4 weeks before pegvisomant treatment. She was then treated with a combination therapy (octreotide LAR + pegvisomant), and after tumor enlargement, pegvisomant was withdrawn, but the tumor continued to grow. The patient was submitted to another surgery and radiotherapy with IGF-I normalization.

Elevated liver enzymes were observed in two patients $(7.4 \%)$ but were mild (1.5 and $2.3 \mathrm{x}$ the ULNR), transient (two and five months) and reverted despite pegvisomant maintenance. Both patients were in combination treatment with pegvisomant and octreotide LAR. No patient presented liver failure or required drug withdrawal due to hepatic side effects.

Lipohypertrophy was observed in two patients (7.4\%); both were non-controlled with pegvisomant therapy and reversed after reeducation regarding the importance of the rotation of the drug injection sites.

Table 1. Comparison of clinical, biochemical and treatment characteristics between patients controlled or not during pegvisomant treatment

\begin{tabular}{lccc}
\hline Variable & Controlled & Not controlled & p-value \\
\hline Age at diagnosis (years) & $36(20-75)$ & $41(28-82)$ & 0.576 \\
Age at the moment of PEG initiation & $47(24-84)$ & $49(36-86)$ & 0.705 \\
Female sex (\%) & 39 & 25 & 1.000 \\
Previous radiotherapy (\%) & 43 & $35.2(15.8-252.0)$ & 0.624 \\
GH levels at diagnosis ( $\mu$ g/L) & $10.6(3.4-198.0)$ & $295(182-322)$ & 0.145 \\
IGF-l levels at diagnosis (\%ULNR) & $300(157-671)$ & $6.5(2.6-209.0)$ & 0.635 \\
GH levels pre-PEG ( $\mu$ g/L) & $2.8(0.7-17.3)$ & $559(214-637)$ & 0.095 \\
IGF-I levels pre-PEG (\%ULNR) & $208(132-390)$ & 0.004 \\
\hline
\end{tabular}

$\%$ ULNR: percentage of the upper limit of normal range. 

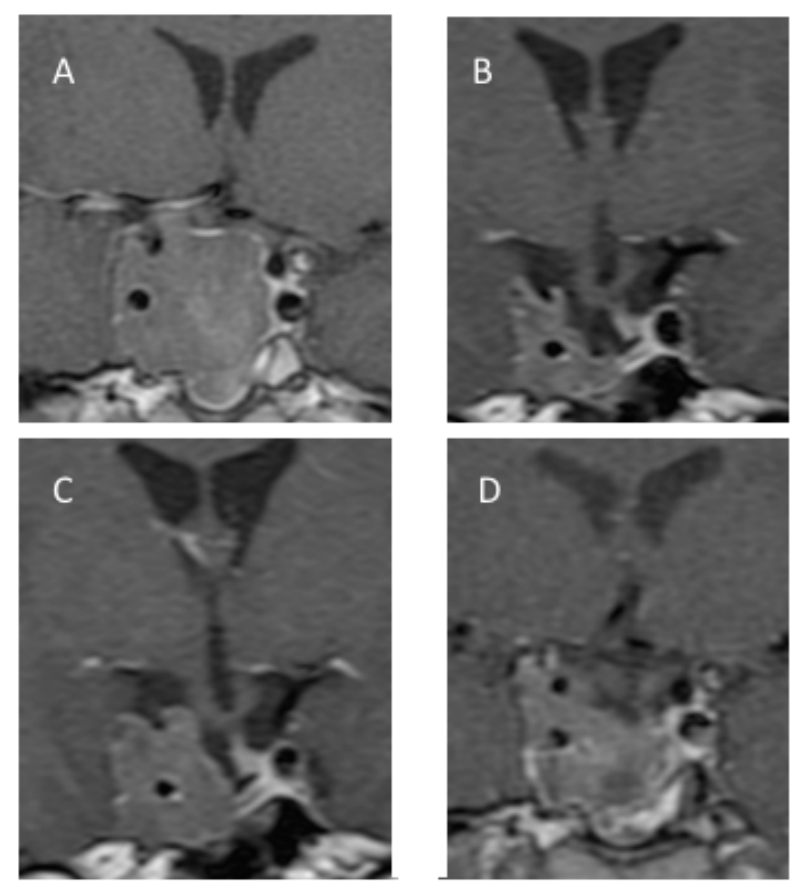

Figure 1. Sellar magnetic resonance imaging ( $\mathrm{T} 1$ weighted after gadolinium enhancement) showing the growth of a residual tumor located mainly in the right cavernous sinus during pegvisomant treatment, that was sustained after drug withdrawal: (A) at diagnosis; (B) before pegvisomant treatment; (C) during pegvisomant treatment; (D) six months after pegvisomant withdrawal.

One patient presented a headache that was possibly related to the drug, as it ceased when the drug was stopped and recurred when pegvisomant was reintroduced, which led to the suspension of the treatment. This patient was being treated with pegvisomant as monotherapy and was the only patient in whom the drug was withdrawn due to a drug-related side effect.

No other drug-related side effects were observed.

\section{DISCUSSION}

We demonstrated that pegvisomant treatment is effective and safe for the first time in a cohort of Brazilian acromegaly patients to accompany descriptions in other populations. Additionally, we explored possible predictors of the treatment response, and only pretreatment IGF-I levels were predictive of disease control with pegvisomant.

Acromegaly is associated with enhanced mortality and a high morbidity when normalization of GH and IGF-I levels is not achieved (2). Although surgery and tumor-directed drugs (SA and DA) permit disease control in the majority of patients, there are some cases that require additional treatments $(4,30)$. In our series, the majority of patients $(78 \%)$ were submitted to surgery, and all but one patient was treated with first-generation SA, with association with cabergoline tried in $78 \%$. No patient received pasireotide LAR. Radiotherapy was administered to $41 \%$ of the patients. However, despite all of these treatments, they maintained elevated GH and IGF-I levels.

Pegvisomant is highly effective in normalizing IGF-I levels, even in patients resistant to other treatments (30), and this was confirmed in our series with a high percentage $(85 \%)$ of disease control. Our results are closer to the data reported in the initial clinical trials with pegvisomant monotherapy and those from series of other tertiary centers than to the data reported in the last update of the Acrostudy (20-22,31,32). One of the possible reasons for a lower control rate in the Acrostudy is the clinical inertia (22). As the databank accepts inclusions from many centers, there are probably some centers with less experience in treating acromegaly and therefore in adjusting treatment to attain the goals of disease control. Because we are a tertiary reference center, drug escalation and the pursuit to obtain disease control is probably more intensive (22). Another possible explanation is the higher number of patients treated with a combination of pegvisomant and SA (74\%) than that observed in the Acrostudy (37\%), as the reported control rates with combination therapy can be as high as $97 \%$ in other studies $(33,34)$. It is also important to highlight that in the Acrostudy, IGF-I normalization was recorded on annual bases, therefore it can provide lower control rates than studies assessing normalization of IGF-I at the last patient visit or at any time during follow-up.

The safety profile of the drug in our patients was also similar to what is reported the literature (22) with only mild elevations of liver enzymes and lipohypertrophy. Only one patient presented tumor growth during treatment, but this patient harbored an aggressive tumor that continued to grow after the drug was withdrawn. There were no cases of serious adverse effects that required suspension of the drug.

There are few studies in the literature addressing predictors of response to pegvisomant treatment $(23,24)$. One of the possible predictors is the presence of the d3GHR isoform of the GHR. Two initial studies, including 19 and 44 patients, described that in the presence of the $\mathrm{d} 3 \mathrm{GHR}$ isoform, a lower dose of pegvisomant and a shorter treatment was necessary 
to obtain normalization of IGF-I levels. However, Filopanti and cols. (27) in a larger multicenter study (111 patients) did not find a difference in the response rates to pegvisomant treatment between those patients presenting d3GHR or the flGHR. Additionally, Franck and cols. (28) recently described no difference in the response rates during combination therapy with pegvisomant and SA in patients presenting the different isoforms of GHR. Our results are in accordance with these larger studies in the literature; we also found no difference in the treatment response rates.

The only predictor of response in our series was the pre-pegvisomant IGF-I level. Although prepegvisomant $\mathrm{GH}$ levels were lower in those patients controlled after pegvisomant treatment than in the noncontrolled patients, the difference was not statistically significant $(\mathrm{p}=0.095)$. As we mentioned, we also did not find a difference in the control rate in the presence of the $\mathrm{d} 3 \mathrm{GHR}$ isoform. However, considering the number of patients in our series, a type II error cannot be excluded.

In conclusion, treatment with pegvisomant was highly effective and safe in our series of Brazilian patients as previously reported in other populations. A better chance of disease control can be expected in those with lower pre-pegvisomant IGF-I levels, and no difference was observed in the presence or absence of the $\mathrm{d} 3 \mathrm{GHR}$ isoform.

Disclosure: MRG has received unrestricted research grants and speaker fees from Novartis, Ipsen and Pfizer and has participated on advisory boards of Novartis and Ipsen. The other authors have nothing to disclose.

\section{REFERENCES}

1. Colao A, Ferone D, Marzullo P, Lombardi G. Systemic complications of acromegaly: epidemiology, pathogenesis, and management. Endocr Rev. 2004;25(1):102-52.

2. Holdaway IM, Bolland MJ, Gamble GD. A meta-analysis of the effect of lowering serum levels of GH and IGF-I on mortality in acromegaly. Eur J Endocrinol. 2008;159(2):89-95.

3. Dekkers OM, Biermasz NR, Pereira AM, Romijn JA, Vandenbroucke JP. Mortality in acromegaly: a metaanalysis. J Clin Endocrinol Metab. 2008;93(1):61-7.

4. Katznelson L, Laws ER Jr, Melmed S, Molitch ME, Murad MH, Utz A, et al. Acromegaly: an endocrine society clinical practice guideline. J Clin Endocrinol Metab. 2014;99(11):3933-51.

5. Nomikos P, Buchfelder M, Fahlbusch R. The outcome of surgery in 668 patients with acromegaly using current criteria of biochemical "cure". Eur J Endocrinol. 2005;152(3):379-87.

6. Melmed S, Colao A, Barkan A, Molitch M, Grossman AB, Kleinberg $D$, et al. Guidelines for acromegaly management: an update. J Clin Endocrinol Metab. 2009;94(5):1509-17.
7. Mercado M, Borges F, Bouterfa H, Chang TC, Chervin A, Farrall AJ, et al. A prospective, multicentre study to investigate the efficacy, safety and tolerability of octreotide LAR (long-acting repeatable octreotide) in the primary therapy of patients with acromegaly. Clin Endocrinol (Oxf). 2007;66(6):859-68.

8. Colao A, Cappabianca P, Caron P, De Menis E, Farrall AJ, Gadelha $M R$, et al. Octreotide LAR vs. surgery in newly diagnosed patients with acromegaly: a randomized, open-label, multicentre study. Clin Endocrinol (Oxf). 2009;70(5):757-68.

9. Melmed S, Cook D, Schopohl J, Goth MI, Lam KS, Marek J. Rapid and sustained reduction of serum growth hormone and insulin-like growth factor- 1 in patients with acromegaly receiving lanreotide Autogel therapy: a randomized, placebo-controlled, multicenter study with a 52 week open extension. Pituitary. 2010;13(1):18-28.

10. Caron PJ, Bevan JS, Petersenn S, Flanagan D, Tabarin A, Prevost G, et al.Tumor shrinkage with lanreotide Autogel $120 \mathrm{mg}$ as primary therapy in acromegaly: results of a prospective multicenter clinical trial. J Clin Endocrinol Metab. 2014;99(4):1282-90.

11. Annamalai AK, Webb A, Kandasamy N, Elkhawad M, Moir S, Khan $F$, et al. A comprehensive study of clinical, biochemical, radiological, vascular, cardiac, and sleep parameters in an unselected cohort of patients with acromegaly undergoing presurgical somatostatin receptor ligand therapy. J Clin Endocrinol Metab. 2013;98(3):1040-50.

12. Gadelha MR, Bronstein MD, Brue T, Coculescu M, Fleseriu M, Guitelman $\mathrm{M}$, et al. Pasireotide versus continued treatment with octreotide or lanreotide in patients with inadequately controlled acromegaly (PAOLA): a randomised, phase 3 trial. Lancet Diabetes Endocrinol. 2014;2(11):875-84.

13. Colao A, Bronstein MD, Freda P, Gu F, Shen CC, Gadelha M, et al. Pasireotide versus octreotide in acromegaly: a head-to-head superiority study. J Clin Endocrinol Metab. 2014;99(3):791-9.

14. Kasuki L, Vieira Neto L, Gadelha MR. Cabergoline treatment in acromegaly: cons. Endocrine. 2014;46(2):220-5.

15. Sandret $L$, Maison $P$, Chanson P. Place of cabergoline in acromegaly: a meta-analysis. J Clin Endocrinol Metab. 2011;96(5):1327-35.

16. Vandeva S, Elenkova A, Natchev E, Kirilov G, Tcharaktchiev D, Yaneva $M$, et al. Treatment outcome results from the Bulgarian Acromegaly Database: adjuvant dopamine agonist therapy is efficient in less than one fifth of non-irradiated patients. Exp Clin Endocrinol Diabetes. 2015;123(1):66-71.

17. Neto LV, Machado Ede O, Luque RM, Taboada GF, Marcondes JB, Chimelli LM, et al. Expression analysis of dopamine receptor subtypes in normal human pituitaries, nonfunctioning pituitary adenomas and somatotropinomas, and the association between dopamine and somatostatin receptors with clinical response to octreotide-LAR in acromegaly. J Clin Endocrinol Metab. 2009;94(6):1931-7.

18. Ferone D, Gatto F, Arvigo M, Resmini E, Boschetti M, Teti C, et al. The clinical-molecular interface of somatostatin, dopamine and their receptors in pituitary pathophysiology. J Mol Endocrinol. 2009;42(5):361-70.

19. Kopchick JJ. Discovery and mechanism of action of pegvisomant. Eur J Endocrinol. 2003;148 Suppl 2:S21-5.

20. Trainer PJ, Drake WM, Katznelson L, Freda PU, Herman-Bonert V, van der Lely AJ, et al. Treatment of acromegaly with the growth hormone-receptor antagonist pegvisomant. $\mathrm{N}$ Engl J Med. 2000;342(16):1171-7.

21. van der Lely AJ, Hutson RK, Trainer PJ, Besser GM, Barkan AL, Katznelson $L$, et al. Long-term treatment of acromegaly with pegvisomant, a growth hormone receptor antagonist. Lancet. 2001;358(9295):1754-9. 
22. van der Lely AJ, Biller BM, Brue T, Buchfelder M, Ghigo E, Gomez R, et al. Long-term safety of pegvisomant in patients with acromegaly: comprehensive review of 1288 subjects in ACROSTUDY. J Clin Endocrinol Metab. 2012;97(5):1589-97.

23. Parkinson C, Burman P, Messig M, Trainer PJ. Gender, body weight, disease activity, and previous radiotherapy influence the response to pegvisomant. J Clin Endocrinol Metab. 2007;92(1):190-5.

24. Sievers C, Baur DM, Schwanke A, Buchfelder M, Droste M, Mann $\mathrm{K}$, et al. Prediction of therapy response in acromegalic patients under pegvisomant therapy within the German ACROSTUDY cohort. Pituitary. 2015;18(6):916-23.

25. Bianchi A, Mazziotti G, Tilaro L, Cimino V, Veltri F, Gaetani E, et al. Growth hormone receptor polymorphism and the effects of pegvisomant in acromegaly. Pituitary. 2009;12(3):196-9.

26. Bernabeu I, Alvarez-Escola C, Quinteiro C, Lucas T, Puig-Domingo $M$, Luque-Ramirez $M$, et al. The exon 3-deleted growth hormone receptor is associated with better response to pegvisomant therapy in acromegaly. J Clin Endocrinol Metab. 2010;95(1):222-9.

27. Filopanti M, Olgiati L, Mantovani G, Corbetta S, Arosio M, Gasco V, et al. Growth hormone receptor variants and response to pegvisomant in monotherapy or in combination with somatostatin analogs in acromegalic patients: a multicenter study. J Clin Endocrinol Metab. 2012;97(2):E165-72.

28. Franck SE, Van der Lely AJ, Delhanty PJ, Jorgensen JO, Neggers SJ. Pegvisomant in combination with long-acting somatostatin analogues in acromegaly: the role of the growth hormone receptor deletion of exon 3. Eur J Endocrinol. 2015;173(5):553-61.
29. Lima GA, Wu Z, Silva CM, Barbosa FR, Dias JS, Schrank Y, et al. Growth hormone isoforms in acromegalic patients before and after treatment with octreotide LAR. Growth Horm IGF Res. 2010;20(2):87-92.

30. Colao A, Pivonello R, Auriemma RS, De Martino MC, Bidlingmaier $M$, Briganti $F$, et al. Efficacy of 12-month treatment with the GH receptor antagonist pegvisomant in patients with acromegaly resistant to long-term, high-dose somatostatin analog treatment: effect on IGF-I levels, tumor mass, hypertension and glucose tolerance. Eur J Endocrinol. 2006;154(3):467-77.

31. Marazuela $M$, Lucas $T$, Alvarez-Escola $C$, Puig-Domingo $M$, de la Torre NG, de Miguel-Novoa $P$, et al. Long-term treatment of acromegalic patients resistant to somatostatin analogues with the $\mathrm{GH}$ receptor antagonist pegvisomant: its efficacy in relation to gender and previous radiotherapy. Eur $\mathrm{J}$ Endocrinol. 2009;160(4):535-42.

32. Cannavo S, Bogazzi F, Colao A, De Marinis L, Maffei P, Gomez $R$, et al. Does pegvisomant treatment expertise improve control of resistant acromegaly? The Italian ACROSTUDY experience. J Endocrinol Invest. 2015;38(10):1099-109.

33. Neggers SJ, Franck SE, de Rooij FW, Dallenga AH, Poublon RM, Feelders RA, et al. Long-term efficacy and safety of pegvisomant in combination with long-acting somatostatin analogs in acromegaly. J Clin Endocrinol Metab. 2014;99(10):3644-52.

34. Neggers SJ, van der Lely AJ. Combination treatment with somatostatin analogues and pegvisomant in acromegaly. Growth Horm IGF Res. 2011;21(3):129-33. 